IRSTI 34.27 .39

\author{
${ }^{1 *}$ A. Kondybayev, A. ${ }^{1}$ Zhakupbekova, ${ }^{1}$ F. Amutova, ${ }^{1}$ A. Omarova, \\ ${ }^{1} \mathrm{M}$. Nurseitova, ${ }^{1}$ Sh. Akhmetsadykova, \\ ${ }^{1,2} \mathrm{~N}$. Akhmetsadykov, ${ }^{1}$ G. Konuspayeva, ${ }^{4}$ B. Faye \\ ${ }^{1}$ ANTIGEN Co. LTD, Almaty region, Azerbayev Str., 4, Abay v., 040905 Kazakhstan \\ ${ }^{3}$ Kazakh National Agrarian University, Abai str., 8, 050010, Almaty, Kazakhstan \\ ${ }^{4}$ UMR Selmet, Cirad, Montpellier, France \\ *e-mail: askond@gmail.com
}

\title{
Volatile organic compounds profiles in milk fermented by lactic bacteria
}

\begin{abstract}
The organoleptic properties of traditional dairy beverages done with non-conventional dairy species (horse, camel), popular in Central Asia, were rarely described in the literature. To characterize the Volatile Organic Compounds (VOC) profile of fermented mare milk, 12 samples of cow milk, used as matrix were inoculated by different strains from two types of bacteria (bacilli and cocci) isolated from natural fermented mare milk. The analysis performed by Gas Chromatography coupled with Solid-Phase Micro-Extraction allowed identification of 160 different compounds from the 12 strains, and 90 from natural fermented mare milk. After cluster analysis, 3 types of profiles were observed. Those profiles were distinct by the amount of acid compounds (low, medium, high), negatively related to aromatic and aliphatic hydrocarbons. The analysis of the mean volatile compound profile of each type of bacteria (bacilli and cocci) by factorial discriminant analysis showed that 3 molecules (oxime-methoxy-phenyl, propanedioic acid propyl and 2-propanamine) allowed to well class $100 \%$ of the samples. Further researches on bacterial identification and experiments with different fermentation matrices from other dairy species will be conducted.
\end{abstract}

Key words: volatile organic compounds, mare milk, discriminant analysis, lactic acid bacteria, fermented milk.

\section{Introduction}

Volatile organic compounds (VOC) are used to characterize the organoleptic properties of dairy products $[1 ; 2]$. However, their routine determination is relatively new due to the improvement of the equipment for their detection. Consequently, the characterization of dairy products by VOCs is also new and provides a better knowledge of the molecules responsible for aroma production in those products. VOCs composition in dairy products depends on several factors: types of feeds of the dairy animal, microflora naturally present in the raw milk, and also on the processing procedure. The taste and smell of dairy products largely depend on the degree of accumulation of volatile carbonyl compounds, carboxylic acids and other aromatic substances. These compounds determine not only the taste and quality of products, but also have great physiological importance, since they contribute to the secretion of digestive juices and provide good digestibility of the product by human.
In Central Asia, the consumption of fermented milk from different dairy species (cow, horse, camel) is an important element of the local culture [3]. Data for describing those local products by their physico-chemical, biochemical and microbiological composition is widely available [4], but the organoleptic properties were defined only by qualitative description. At our knowledge, the characterization of organoleptic properties of fermented mare milk (koumiss), one of the typical products produced in Kazakhstan, by the analysis of VOCs was never achieved. Microorganisms responsible of fermentation process are involved in the production of a large number of different volatile compounds, including, alcohols, esters, hydrocarbons, terpenes, ketones, sulfur-containing compounds and carboxylic acids.

Thus, the objective of the present paper was to determine the VOC profile of milk fermented by different microorganisms isolated from mare milk.

Printed in Kazakhstan 


\section{Materials and methods}

Samples used. Five samples of fermented mare milk (koumis) and one sample of raw mare milk from 6 different locations of Kazakhstan (Merke, Almaty, Semey, Taraz, Shymkent and Urzhar) were collected directly on farm in sterile tubes of $10 \mathrm{ml}$. They were carried at $4^{\circ} \mathrm{C}$ to ANTIGEN laboratory for further analysis. The strains of lactic acid bacteria used as starter cultures were isolated from mare's milk and koumiss obtained by spontaneous fermentation and identified as the predominant species during the production process. LAB strains were isolated from sample by using wire loops on the M17 and MRS agar (Biokar Diagnostics, France). After the incubation period $\left(48 \mathrm{~h}, 37^{\circ} \mathrm{C}\right)$, single colonies that had different morphological traits were sub-cultured. Microorganisms were maintained at $-4^{\circ} \mathrm{C}$ in the tubes on the correspondent nutritive medium and at $-20^{\circ} \mathrm{C}$ in the culture broth supplemented with $30 \%$ glycerol. 12 strains of lactic acid bacteria were obtained, including, seven strains of bacilli and five strains of cocci were identified morphologically. The strains were characterized by using Gram's staining (reagent kit "Color Gram2E" BioMérieux, France), catalase tests (ID color catalase ID-ASE Biomérieux, France) and oxidase tests (Oxidase reagent Biomérieux, France).

Experiment design. One unique matrix (commercial UHT cow milk Lactel $^{\circ}, 2.5 \%$ fat matter) was used for inoculation of each specific strain for fermentation process. Normalized cow milk was heated to $65^{\circ} \mathrm{C}$, homogenized at $20 \mathrm{MPa}$, pasteurized at $95^{\circ} \mathrm{C}$ for $5 \mathrm{~min}$ and cooled to inoculation temperature. Milk was inoculated (5\% of the mother culture) and incubated at $37^{\circ} \mathrm{C}$. Samples of this matrix without inoculation were used as control.

After inoculation, samples were stored at $37^{\circ} \mathrm{C}$ for 48 hours to start the fermentation process. Then, each sample was analyzed for VOCs profiles determination using Gas Chromatography with Mass spectrometry detection (GC-MS). For VOCs profile determination, one control was tested for each day of analysis (3 controls). One sample of naturally fermented koumis from Taraz was also analyzed for comparison.

Analytical procedure. Solid-phase microextraction (SPME) technique with $50 / 50 \mu \mathrm{m} \mathrm{DVB} / \mathrm{CAR} /$ PDMS extraction fiber was used for sample preparation according to method of Xu et al. [5]. The extraction temperature was $60^{\circ} \mathrm{C}$, extraction time $30 \mathrm{~min}$ utes, the depth of fiber immersion in the vial $22 \mathrm{~mm}$. The desorption time was $3 \mathrm{~min}$.
After the extraction, the fiber was placed in the injector of gas chromatograph, heated to $260^{\circ} \mathrm{C}$ in splitless mode. Separation of VOC was carried out using a $30 \mathrm{~m}$ long capillary column HP-5MS (Agilent, USA), inner diameter $-0.25 \mathrm{~mm}$ and a film thickness of $0.25 \mu \mathrm{m}$ at a constant gas carrier (helium) rate of $1.0 \mathrm{ml} / \mathrm{min}$.

The chromatography temperature was programmed from $40^{\circ} \mathrm{C}(5 \mathrm{~min})$ to $200^{\circ} \mathrm{C}$ at a heating rate of $5^{\circ} \mathrm{C} / \mathrm{min}(5 \mathrm{~min})$, followed by heating to $260^{\circ} \mathrm{C}(1 \mathrm{~min})$ at $5^{\circ} \mathrm{C} / \mathrm{min}$. The total chromatographic time was 55 minutes. The temperatures of the interface, ion source and quadrupole of the mass spectrometric detector were 260,230 and $150^{\circ} \mathrm{C}$, respectively.

Mass spectrometric detection was performed in the SCAN mode in the range of mass numbers $(\mathrm{m} / \mathrm{z})$ from 34 to $550 \mathrm{amu}$ (atomic mass unit).

The analysis was carried out on a gas chromatograph with mass spectrometer detector 7890B 15977B quadrupole, with electron impact ionization (Agilent, USA, 2017). The device is annually tested. The chromatograph is equipped with an autosampler MultiPurpose Sampler MPS (Gerstel, Germany, 2017), which allows to automate the analysis of samples. The MassHunter GC/MS Acquisitions B.07.05.2479 and Agilent MSD ChemStation software (version F.01.03.2357) were used to control the gas chromatographic system, record and process the chromatographic data. Data processing included determining retention times, peak heights and areas, and processing of spectral information obtained with the mass spectrometric detector. To investigate the obtained mass spectra, the library Wiley 10th edition was used (the total number of spectra in the library is more than 550,000).

The content of the components was determined by the method of rationing peak areas (reduction to $100 \%$ ), i.e. finding the percentage of each component in the mixture being analyzed.

Statistical analyses. The software used was XLstat, $2017\left(\right.$ Addinsoft $\left.^{\circ}\right)$. The objective of the statistical analysis was to identify VOC profiles according to the different strains used. For that, a multivariate analysis was applied. The raw data table (specific fermented milk samples * percentage of different volatiles compounds) was submitted to Principal Components Analysis (PCA) in order to identify the dissimilarity between the profiles [6].

The type of lactic bacteria was used as supplementary variables to identify the proximity with specific VOCs profiles. In a second step, to simplify 
the reading of the results, all the volatile compounds were grouped into nine organic chemical classes: aliphatic hydrocarbons, aromatic hydrocarbons, alcohols, ketones, aldehydes, acids, esters, nitrogen compounds and others. Such classification was similar to that proposed in the literature [7]. The PCA of the group of compounds was followed by a cluster analysis (Ascending Hierarchical Classification - AHC) on Ward method to identify groups of profiles [8].

To assess the difference between the two types of lactic acid bacteria (bacilli and cocci), a multivariate discriminant analysis (MDA) with forward stepwise model was applied [9]. Then, due to non-normalized data, non-parametric Mann-Whitney test was achieved to assess the significant level of difference between the VOCs profiles of these two lactic acid bacteria. Discriminant analysis was applied both on VOCs group and on VOCs profiles.

\section{Results and discussion}

As the whole, 160 volatile compounds were identified. The list of the molecules reported in Table 1 was grouped by class of organic compounds. The group "hydrocarbons" included aliphatic hydrocarbons $(n=31)$ and aromatic hydrocarbons represented by only one compound (benzene, 1,3-bis(1,1-dimethylethyl). The number of molecules differed between samples: 14 molecules in sample 2; 16 in sample 3; 28 in sample $4 ; 17$ in sample $5 ; 17$ in sample $6 ; 29$ in sample 7; 12 in sample 8; 25 in sample 9; 27 in sample 10; 47 in sample 11;29 in sample 12 and 49 in sample 13. The controls contained 13 molecules only.

In comparison, the natural koumis contained 90 different molecules and only 5 were common to the specific fermented milk (Table 2): decanoic acid, ethyl ester (25.7\%), ethanol (5.8\%), octanoic acid (2.07\%), dodecanoic acid (1.57\%), 2-methyl-5H-dibenz[b,f] azepine $(0.12 \%)$ and nonanoic acid $(0.02 \%)$.

In order to facilitate the analysis, a table including our 13 samples (including the 3 controls) described by the sum of percentage for each class of molecules (9 groups) was created and submitted to Principal Components Analysis.

Analysis of the groups of VOCs. The main factors of the PCA explained almost $60 \%$ of the variance and are marked by the opposition between profiles rich in hydrocarbons at the right side of the factorial plan, and profiles rich in acids and nitrogen-compounds at the left side. On the second factor, the variable "others" was the main contributive variables with aldehydes in opposition to profiles rich in alcohols (Figure 1).

The cluster analysis showed 3 main groups of VOCs profiles (Figure 2) with high difference in their main composition (Figure 3 ).

The type 1 (samples 2, 7, 12, controls C1, C2 and C3) were composed mainly by aliphatic, aromatic and ketone compounds. The second type which contained 6 samples (numbers 3, 4, 8, 9, 10, 11, and 13) was characterized by its richness in ketones and acids. The last type (samples 5 and 6 ) contained mostly acid compounds (90\%).

Description of the samples by their full profile (Figure $4 a, b$ and $c$ ). Each sample was described by the proportion of each group of VOCs sorted, in the order, in acids (AC), aldehydes (AD), alcohols (AH), nitrogen-compounds (AM), esters (ES), aliphatic hydrocarbons (HCF), aromatic hydrocarbons (HCR), ketones (KE) and others (OT). In addition to the main components described above by groups, the analysis by molecule showed that the type 1 contained a small part of alcohol compounds ( $5 \%$ on average) and the type 2 a highest quantity of $\mathrm{N}$-compounds, esters and others compounds while in type 3 , only VOCs from acid groups were predominant.

The analysis of the table (13 samples*160 VOCs) by PCA showed that 3 samples had specific profiles quite different than the others. The sample 13 was the most original product with 49 compounds and was the only sample containing ethylene oxide, 3,5-dichloro-2-hydroxybenzaldehyde, 5,5-d2trans-3,4-dihydroxy-cyclope, 2-pentanamine, 3-amino-2-methylbutanoic acid, 12-methylaminolauric acid, ethanol, 2-(ethenyloxy), butanoic acid and methyl ester. After discarding sample 13, the most original sample was $n^{\circ} 11$ which is the only sample containing many molecules, especially ethanamine N-methyl-, cyclobutanol, 2,3-butanedione, acetic formic anhydride, 5-trideuteromethyltetrazole, benzeneethanamine, 2 -fluoro- $\beta, 5$-dihydroxy$\mathrm{N}$-methyl-, butanoic acid, butane, 1,2,4-trimethoxy-, 2,4-Pentanediol, and 2-methyl- representing as the whole $34 \%$ of the molecules in the sample. The sample $\mathrm{n}^{\circ} 7$ was alone to contain hexane, 2,3,5-trimethyl, 1,3-dimethylthioindole, 1H-Imidazole, 2-nitro, methyltetradecan-2-ol 2-, alpha-DGlucopyranoside, beta.-, nonanal, 1,3-dioxolane, 2-ethyl-2-methyl-, and heptanoic acid. 
Table 1 - List of the volatile organic compounds by class of molecules identified in fermented milk samples

\begin{tabular}{|c|c|c|c|c|c|c|c|}
\hline Acids & Aldehydes & Alcohol & N Compounds & Esters & Hydrocarbones & Ketones & Others \\
\hline $\begin{array}{l}\text { 12-Methylami- } \\
\text { nolauric Acid; } \\
\text { 2-Amino- } \\
\text { 6-Methylbenzo- } \\
\text { ic Acid; } \\
\text { Acetic Acid; } \\
\text { Acetic Acid, } \\
\text { Sodium Salt; } \\
\text { Acetic Formic } \\
\text { Anhydride; } \\
\text { Benzoic Acid; } \\
\text { Butanoic Acid; } \\
\text { Butanoic Acid, } \\
\text { 2-Methyl-; }\end{array}$ & $\begin{array}{l}\text { 2-Butenal, } \\
\text { 3-Methyl-; } \\
\text { 3,5-Dichloro- } \\
\text { 2-Hydroxybenz- } \\
\text { aldehyde; } \\
\text { Acetaldehyde; } \\
\text { Benzaldehyde, } \\
\text { 2-Methyl-; } \\
\text { Benzaldehyde, } \\
\text { 3-Methyl-; } \\
\text { Benzaldehyde, } \\
\text { 4-Methyl-; } \\
\text { Butanal; } \\
\text { Dodecanal; } \\
\text { Hexanal; } \\
\text { Nonanal; }\end{array}$ & $\begin{array}{l}\text { (S)-(+)-6-Methyl- } \\
\text { 1-Octanol; } \\
\text { 1-(Benzylsulfa- } \\
\text { nyl)Pentan-2-Ol; } \\
\text { 1H-Imidazole, } \\
\text { 2-Nitro-; } \\
\text { 2,4-Pentanediol, } \\
\text { 2-Methyl-; } \\
\text { 2-Butanol, } \\
\text { 3-Methyl-; }\end{array}$ & $\begin{array}{l}\text { 1,3-Dimethylthio- } \\
\text { indole; } \\
\text { 1,4-Dinitrotetrahy- } \\
\text { droimidazo [4, 5-D; } \\
\text { 1-Amido-1-Cyano- } \\
\text { 3-Methylbut-1-Ene; } \\
\text { 1-Butanamine, N- } \\
\text { Methyl-; } \\
\text { 2-Butanamine; } \\
\text { 2-Butanamine, } \\
\text { 3-Methyl-; }\end{array}$ & $\begin{array}{l}\text { Butane, 1,2,4-Tri- } \\
\text { methoxy-; } \\
\text { Butanoic Acid, } \\
\text { Methyl Ester; } \\
\text { Carbamic Acid, } \\
\text { Methyl Ester; } \\
\text { Decanoic Acid, } \\
\text { Ethyl Ester; } \\
\text { Formic Acid, } \\
\text { 3-Methylbut-2-Y1 } \\
\text { Este; }\end{array}$ & $\begin{array}{l}\text { (2e)-3-Methyl- } \\
\text { 2-Pentene; } \\
\text { (3e)-2-Methyl- } \\
\text { 3-Heptene; } \\
\text { (4e)-4-Methyl- } \\
\text { 4-Decene; } \\
\text { 1,1'-Bicyclo- } \\
\text { hexyl, 2-Ethyl-, } \\
\text { Trans; } \\
\text { 1-Dodecene; } \\
\text { 1-Undecene; } \\
\text { 2,4-Dimethyl- } \\
\text { 1-Heptene; } \\
\text { 2-Pinene; } \\
\text { 5-Dodecene, } \\
\text { (E)-; }\end{array}$ & $\begin{array}{l}\text { 2,3-Butanedione; } \\
\text { 2-Butanone, } \\
\text { 3-Hydroxy-; } \\
\text { 2-Heptanone; } \\
\text { 2-Heptanone, } \\
\text { 4,6-Dimethyl-; } \\
\text { 2-Heptanone, } \\
\text { 4-Methyl-; } \\
\text { 2-Hexanone, } \\
\text { 5-Methyl-; }\end{array}$ & $\begin{array}{l}\text { (1s)-2,6,6- } \\
\text { Trimethylbicyc- } \\
\text { lo[3.1.1]; } \\
\text { 1,3-Dioxolane, } \\
\text { 2-Ethyl-2-Meth- } \\
\text { yl-; } \\
\text { 1- [2,5 - Dime- } \\
\text { thoxy-4 - (Meth- } \\
\text { ylsulfonyl; } \\
\text { 2-Mercapto - } 4 \text { - } \\
\text { Phenylthiazole; } \\
\text { 5,5-D2-Trans- 3, } \\
\text { 4-Dihydroxy- } \\
\text { Cyclope; }\end{array}$ \\
\hline $\begin{array}{l}\text { Butanoic Acid, } \\
\text { 3-Methyl-; } \\
\text { Carbonic Acid, } \\
\text { Hexyl Prop-1- } \\
\text { En-2-Yl Ester; } \\
\text { Decanoic Acid; } \\
\text { Diglycolic } \\
\text { Acid, Isohexyl } \\
\text { 2-Methyl; } \\
\text { Dodecanoic } \\
\text { Acid; } \\
\text { Heptanoic Acid; } \\
\text { Hexanoic Acid; } \\
\text { Hexanoic Acid, } \\
\text { 2-Methyl-; } \\
\text { L) 3-Amino-2- } \\
\text { Methylbutanoic } \\
\text { Acid; } \\
\text { N-Decanoic } \\
\text { Acid; } \\
\text { Nonanoic Acid; } \\
\text { Octanoic Acid; } \\
\text { Pentanoic Acid; } \\
\text { Pentanoic Acid, } \\
\text { 3-Methyl-; } \\
\text { Propanedioic } \\
\text { Acid, Propyl-; } \\
\text { Propanoic Acid, } \\
\text { 2-Methyl-, } \\
\text { Anhydri; }\end{array}$ & & $\begin{array}{l}\text { 2-Heptanol; } \\
\text { 2-Methyl-2-Dec- } \\
\text { anol; } \\
\text { 2-Methyltetra- } \\
\text { decan-2-Ol; } \\
\text { 2-Nonanol; } \\
\text { 3-Buten-2-Ol, } \\
\text { 2-Methyl-; } \\
\text { 3-Heptanol; } \\
\text { 3-Heptanol, } \\
\text { 4-Methyl-; } \\
\text { 4-Heptanol, } \\
\text { 2,6-Dimethyl-; } \\
\text { Cyclobutanol; } \\
\text { D-Gala-L-Ido- } \\
\text { Octitol; } \\
\text { Ethanol; } \\
\text { Ethanol, } \\
\text { 2-(Ethenyloxy)-; } \\
\text { Methyltetradec- } \\
\text { an-2-Ol 2-; } \\
\text { Nonanol, Tri- } \\
\text { methyl-; }\end{array}$ & 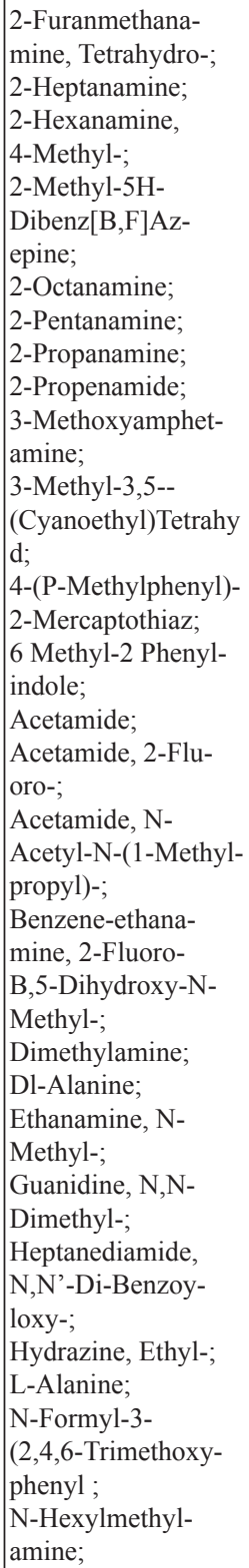 & $\begin{array}{l}\text { Hydrazine, } \\
\text { Methyl-, Oxalate } \\
(1: 1) ; \\
\text { L-Alanine, Ethyl } \\
\text { Ester; } \\
\text { Oxalic Acid, } \\
\text { Hexyl Neopentyl } \\
\text { Ester; } \\
\text { Propane, 2-Eth- } \\
\text { oxy-2-Methyl-; } \\
\end{array}$ & $\begin{array}{l}\text { Cyclohexane, } \\
\text { 1,3,5-Trimethyl-; } \\
\text { Cyclohexane, } \\
\text { 3-Ethyl-5-Meth- } \\
\text { yl-1-Pr; } \\
\text { Cyclopentane, } \\
\text { 1,3-Dimethyl-, } \\
\text { Cis-; } \\
\text { Cyclopropane, } \\
\text { 1,2-Dibutyl-; } \\
\text { Cyclopropane, } \\
\text { 1-Butyl-2-(2- } \\
\text { Methylp; } \\
\text { Decane; } \\
\text { Decane, 4-Meth- } \\
\text { yl-; } \\
\text { Dodecane; } \\
\text { Dodecane, } \\
\text { 2-Methyl-; } \\
\text { Heptane, } \\
\text { 2,2,4-Trimethyl-; } \\
\text { Heptane, 2,4-Di- } \\
\text { methyl-; } \\
\text { Heptane, 4-Meth- } \\
\text { yl-; } \\
\text { Hexane, } \\
\text { 2,2,5-Trimethyl-; } \\
\text { Hexane, } \\
\text { 2,3,5-Trimethyl-; } \\
\text { N-Hexane; } \\
\text { Nonane, 2,6-Di- } \\
\text { methyl- ; } \\
\text { Nonane, 4-Meth- } \\
\text { yl-; } \\
\text { Octane, 3,3-Di- } \\
\text { methyl-; } \\
\text { Pentane, } \\
\text { 2,3,3-Trimethyl-; } \\
\text { Pentane, 2-Meth- } \\
\text { yl-; } \\
\text { Propene 3,3,3- } \\
\text { D3; } \\
\text { R(-)3,7-Dimeth- } \\
\text { yl-1,6-Octadiene; } \\
\text { Benzene, } \\
\text { 1,3-Bis(1,1- } \\
\text { Dimethylethyl; }\end{array}$ & $\begin{array}{l}\text { 2-Hexanone, } \\
\text { 5-Methyl-5-Ni- } \\
\text { tro-; } \\
\text { 2-Nonanone; } \\
\text { 2-Pentanone; } \\
\text { 2-Pentanone, } \\
\text { 4-Hydroxy- } \\
\text { 4-Methyl-; } \\
\text { 2-Propanone; } \\
\text { 2-Undecanone; } \\
\text { Acetone; } \\
\text { Acetophenone; } \\
\text { Ethanone, 1-Cy- } \\
\text { clopentyl-; }\end{array}$ & $\begin{array}{l}\text { 5-Trideuterome- } \\
\text { thyltetrazole; } \\
\text { 6-Chloro-2-(2,6- } \\
\text { Dimethylphenyl) } \\
\text { Qui; } \\
\text { Alpha.-D- } \\
\text { Glucopyranoside, } \\
\text {.Beta.-; } \\
\text { Beta.-D-Gluco- } \\
\text { pyranose, 4-O-. } \\
\text { Beta.; } \\
\text { Bicyclo[3.1.1] } \\
\text { Hept-2-Ene, } \\
\text { 2,6,6-Trimethyl-; } \\
\text { Cyclohex-1,4,5- } \\
\text { Triol-3-One- } \\
\text { 1-Carbo; } \\
\text { Dimethyl Trisul- } \\
\text { fide; } \\
\text { Ethylene Oxide; } \\
\text { Furan, Tetra- } \\
\text { hydro-2,2,5,5- } \\
\text { Tetramet; } \\
\text { Methane, Sulfo- } \\
\text { nylbis-; } \\
\text { Oxime-, Me- } \\
\text { thoxy-Phenyl-; } \\
\text { Oxirane, 2,3-Di- } \\
\text { methyl-, Cis-; } \\
\text { TATP; } \\
\text { Thiazole, 2,5-Di- } \\
\text { methyl }\end{array}$ \\
\hline
\end{tabular}


Table 2 - List of the volatile organic compounds identified in koumiss

\begin{tabular}{|c|c|c|c|c|c|}
\hline Compounds & CAS\# & Area $\%$ & Compounds & CAS\# & Area $\%$ \\
\hline Indene & 000095-13-6 & 0.04 & 1-methyl-1H-indene & 000767-59-9 & 0.01 \\
\hline Toluene & $000108-88-3$ & 0.02 & 9-Hexadecenoic acid, methyl ester, & 001120-25-8 64 & 0.10 \\
\hline Ethanol & $000064-17-5$ & 5.80 & Ethyl 3-phenylpropionate & $002021-28-5$ & 0.36 \\
\hline L-Lactic acid & 000079-33-4 & 1.38 & Octanoic acid, 3-methylbutyl ester & 002035-99-6 & 0.07 \\
\hline Phenol, 2-methoxy- & 000090-05-1 & 0.10 & Pentadecanoic acid, 3-methylbutyl & 002306-91-4 & 0.04 \\
\hline Naphthalene & 000091-20-3 & 0.33 & 2-methoxy-4-(n-propyl)phenol & $002785-87-7$ & 0.15 \\
\hline Naphthalene, 2-methyl- & $000091-57-6$ & 0.02 & Guaiacol, 4-ethyl- & 002785-89-9 & 0.24 \\
\hline Benzoic acid, ethyl ester & 000093-89-0 & 0.01 & Dodecanoic acid, propyl ester & $003681-78-5$ & 0.09 \\
\hline o-Cresol & $000095-48-7$ & 0.01 & Benzofuran, 2-methyl- & $004265-25-2$ & 0.05 \\
\hline $\begin{array}{c}\text { Propanoic acid, 2-hydroxy-, } \\
\text { ethyl ester }\end{array}$ & 000097-64-3 & 0.20 & D-Limonene & $005989-27-5$ & 0.45 \\
\hline $\begin{array}{l}\text { 1,4-Cyclohexadiene, } \\
\text { 1-methyl-4-(1- }\end{array}$ & $000099-85-4$ & 0.10 & (E)-9-Octadecenoic acid ethyl este & 006114-18-7 & 0.05 \\
\hline $\begin{array}{c}\text { Benzene, 1-methyl-4-(1- } \\
\text { methylethyl }\end{array}$ & 000099-87-6 & 0.04 & Isoamyl laurate & $006309-51-9$ & 0.01 \\
\hline Benzene, ethenyl- & $000100-42-5$ & 0.04 & Benzenebutanoic acid, ethyl ester & 010031-93-3 & 0.03 \\
\hline Benzonitrile & 000100-47-0 & 0.02 & 2,6-Bis(1,1-dimethylethyl)-4-(1-ox & 014035-34-8 & 0.03 \\
\hline Benzenemethanol & 000100-51-6 & 0.10 & Tetradecanoic acid, propyl ester & $014303-70-9$ & 0.01 \\
\hline Benzaldehyde & $000100-52-7$ & 0.01 & 9-Decenoic acid & 014436-32-9 & 0.04 \\
\hline $\begin{array}{c}\text { Acetic acid, phenyl-, ethyl } \\
\text { ester }\end{array}$ & 000101-97-3 & 0.02 & Benzofuran, 7-methyl- & $017059-52-8$ & 0.03 \\
\hline Butanoic acid, ethyl ester & $000105-54-4$ & 0.21 & Ethyl tridecanoate & 028267-29-0 & 0.03 \\
\hline Heptanoic acid, ethyl ester & 000106-30-9 & 0.02 & $\mathrm{n}$-capric acid isobutyl ester & $030673-38-2$ & 0.03 \\
\hline Octanoic acid, ethyl ester & 000106-32-1 & 12.57 & Decanoic acid, propyl ester & 030673-60-0 & 0.22 \\
\hline Dodecanoic acid, ethyl ester & 000106-33-2 & 0.03 & $\begin{array}{c}\text { 1H-Isoindole-1,3(2H)-dithione, } \\
\text { 2-ethyl- }\end{array}$ & 035373-06-9 & 0.55 \\
\hline Dodecanoic acid, ethyl ester & 000106-33-2 & 17.25 & Pentadecanoic acid, ethyl ester & 041114-00-5 & 0.13 \\
\hline Phenol & $000108-95-2$ & 0.07 & Methyl(methyl 2,3,4-tri-O-methyl- & $052729-97-2$ & 0.03 \\
\hline Decanoic acid, ethyl ester & 000110-38-3 & 25.68 & Ethyl 9-hexadecenoate & $054546-22-4$ & 0.65 \\
\hline Decanoic acid, methyl ester & $000110-42-9$ & 0.02 & Ethyl 9-hexadecenoate & $054546-22-4$ & 0.01 \\
\hline Dodecanoic acid, methyl ester & $000111-82-0$ & 0.03 & Ethyl 9-hexadecenoate & $054546-22-4$ & 1.37 \\
\hline Nonanoic acid & 000112-05-0 & 0.02 & Butanal, 2,3,4-tris[(trimethylsily & 056196-36-2 & 0.04 \\
\hline Phenol, 4-ethyl- & 000123-07-9 & 0.03 & L-Phenylalanine, N-(trifluoroacety & $058072-44-9$ & 0.01 \\
\hline Nonanoic acid, ethyl ester & $000123-29-5$ & 0.07 & Ethyl 9-decenoate & $067233-91-4$ & 12.33 \\
\hline Hexanoic acid, ethyl ester & 000123-66-0 & 0.03 & Ethyl (2-hydroxyphenyl)acetate, TM & $067903-47-3$ & 0.08 \\
\hline Hexanoic acid, ethyl ester & 000123-66-0 & 0.97 & 2-Pentanol, 3-chloro-4-methyl-, (R & $074685-48-6$ & 0.04 \\
\hline Tetradecanoic acid, ethyl ester & 000124-06-1 & 0.18 & Decanoic acid, 5-chloro-, chlorome & $080418-82-2$ & 0.11 \\
\hline Tetradecanoic acid, ethyl ester & 000124-06-1 & 6.71 & Octadecanoic acid, ethyl ester & $111-61-5$ & 0.08 \\
\hline Tetradecanoic acid, ethyl ester & 000124-06-1 & 2.70 & Ethyl (S)-(-)-lactate & $2000025-88-4$ & 0.54 \\
\hline Octanoic acid & 000124-07-2 & 2.07 & 2-Methyl-5H-dibenz[b,f]azepine & $2000224-69-5$ & 0.12 \\
\hline Isopropyl palmitate & 000142-91-6 & 0.03 & Glycolic acid-D2-O-(trimethylsilyl & 2000264-93-4 & 0.02 \\
\hline Dodecanoic acid & $000143-07-7$ & 1.57 & 1,4-diphenylbut-3-ene-2-ol & $2000280-70-5$ & 0.10 \\
\hline
\end{tabular}


Continuation of table 2

\begin{tabular}{|c|c|c|c|c|c|}
\hline Compounds & CAS\# & Area\% & Compounds & CAS\# & Area $\%$ \\
\hline Phenol, 4-methoxy- & $000150-76-5$ & 0.02 & Ethyl 9-tetradecenoate & $2000381-02-2$ & 0.77 \\
\hline Benzofuran & $000271-89-6$ & 0.15 & Ethyl 13-methyl-tetradecanoate & $2000434-94-9$ & 0.06 \\
\hline Tetradecanoic acid & $000544-63-8$ & 0.04 & Ethyl 4,8,12-trimethyl-tridecanoat & $2000480-94-9$ & 0.01 \\
\hline Octadecane & $000593-45-3$ & 0.02 & Oleic Acid & $52355-42-7$ & 0.07 \\
\hline Propyl octanoate & $000624-13-5$ & 0.12 & Octanoic acid, 2-butyl ester & $5458-61-7$ & 1.45 \\
\hline Undecanoic acid, ethyl ester & $000627-90-7$ & 0.02 & $\begin{array}{c}\text { [1, '-Bicyclopropyl]-2-octanoic acid, } \\
\text { 2'-hexyl-, methyl ester }\end{array}$ & $56687-68-4$ & 0.02 \\
\hline Undecanoic acid, ethyl ester & $000627-90-798$ & 0.12 & $\begin{array}{c}\text { 2-(1-Methyl-1-silacyclobutyl)benzoic } \\
\text { acid trimethyl-silyl ester }\end{array}$ & & 0.17 \\
\hline Hexadecanoic acid, ethyl ester & $000628-97-7$ & 0.01 & 3-Trifluoroacetoxypentadecane & & 0.05 \\
\hline Pentadecane & $000629-62-9$ & 0.05 & E-11-Hexadecenoic acid, ethyl ester & & 0.04 \\
\hline Heptadecane & $000629-78-7$ & 0.04 & & & \\
\hline
\end{tabular}

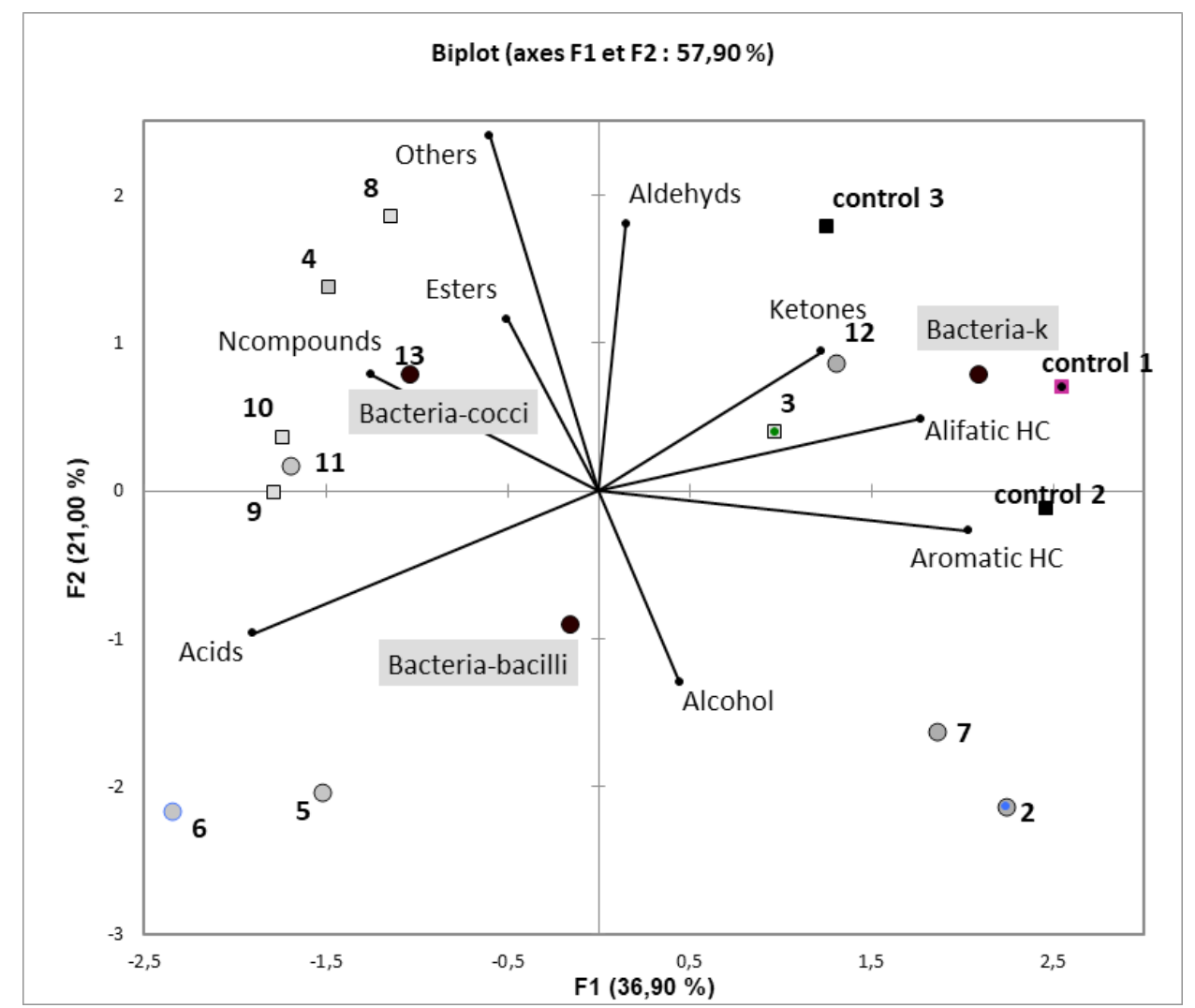

Figure 1 - Factorial plan (f1, f2) of the Principal Components Analysis (biplot projection) showing the projection of active variables (volatile compounds), supplementary variables

(bacteria strains) and fermented milk samples inoculated with bacilli (०) or cocci (口) 


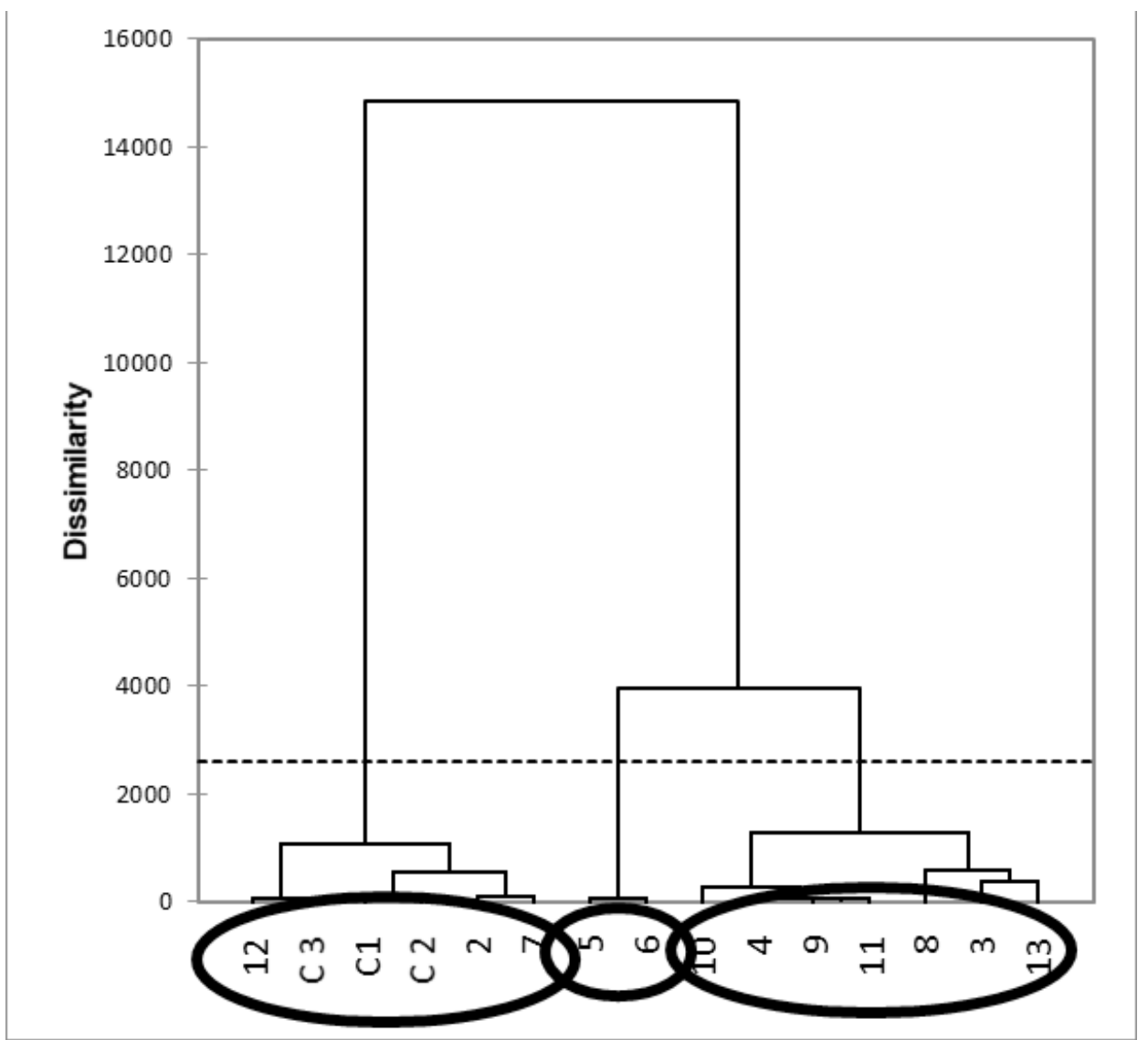

Figure 2 - Dissimilarity based classification tree of the 13 fermented milk samples and control samples $(\mathrm{C} 1, \mathrm{C} 2$ and $\mathrm{C} 3)$ according to their VOCs composition

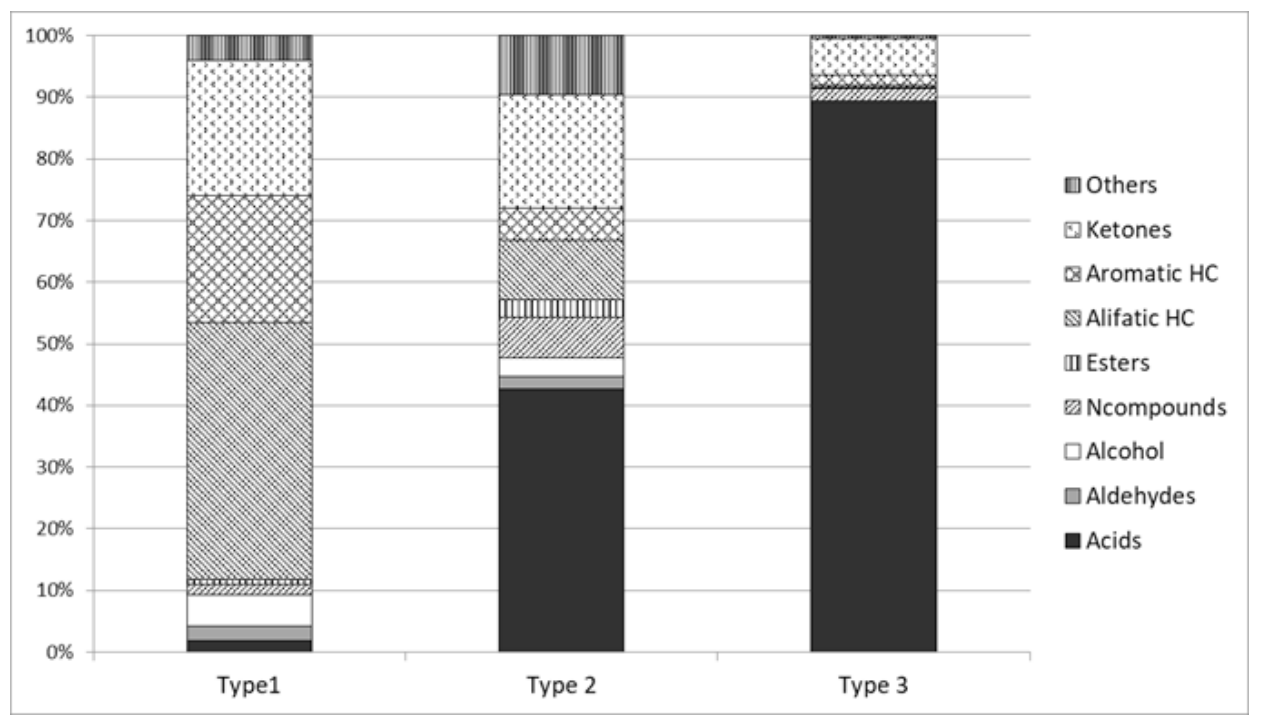

Figure 3 - Mean composition of VOCs classes in each type of samples obtained from classification tree 

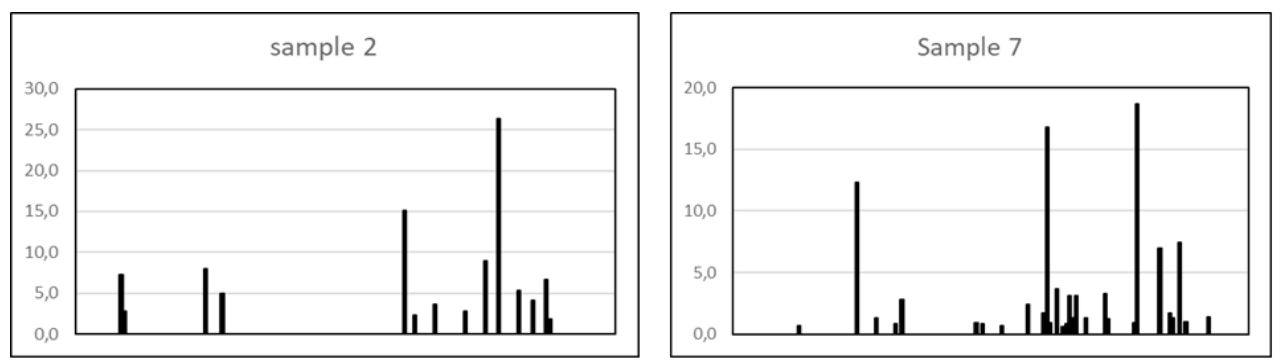

A
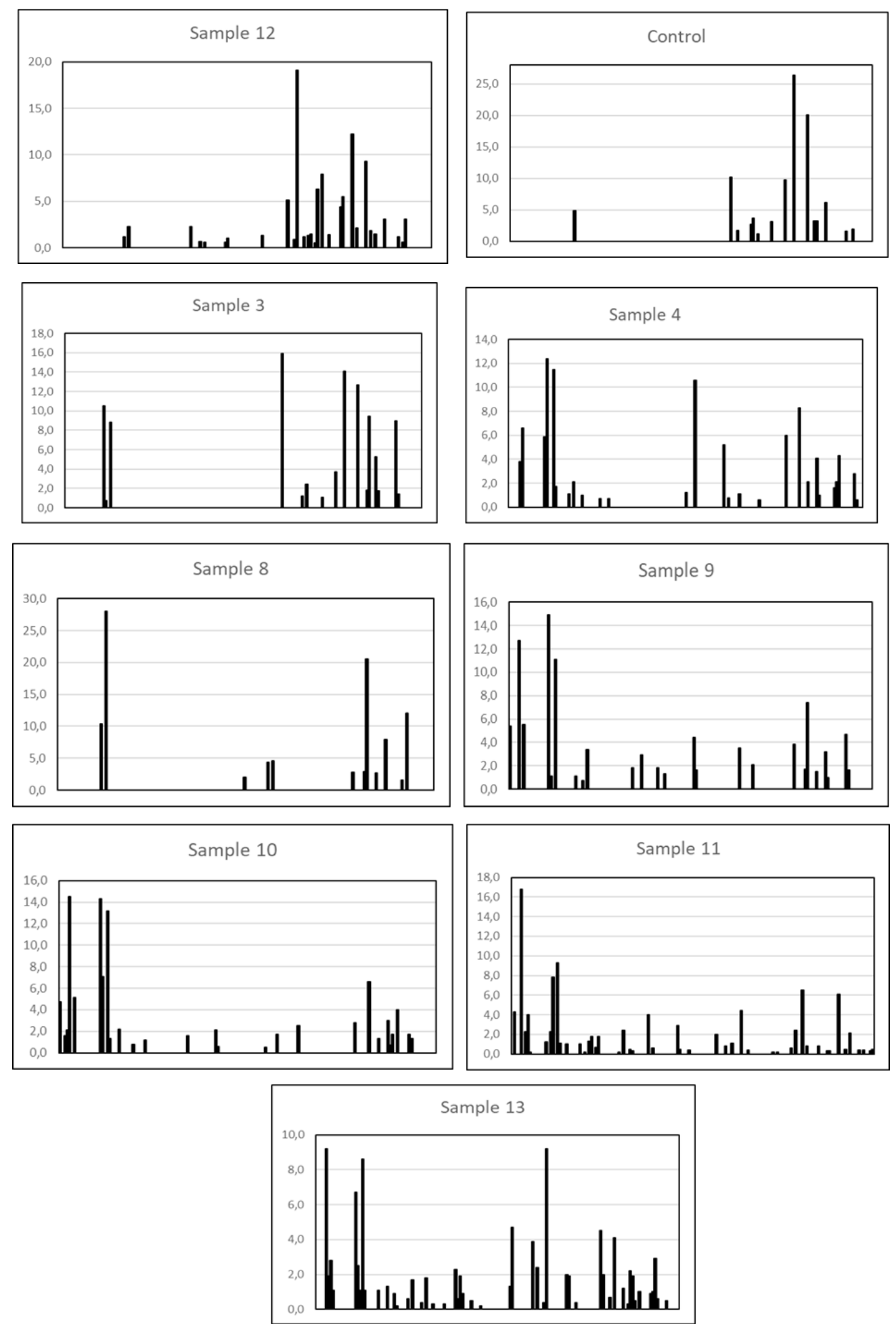

International Journal of Biology and Chemistry 11, № 2, 57 (2018) 

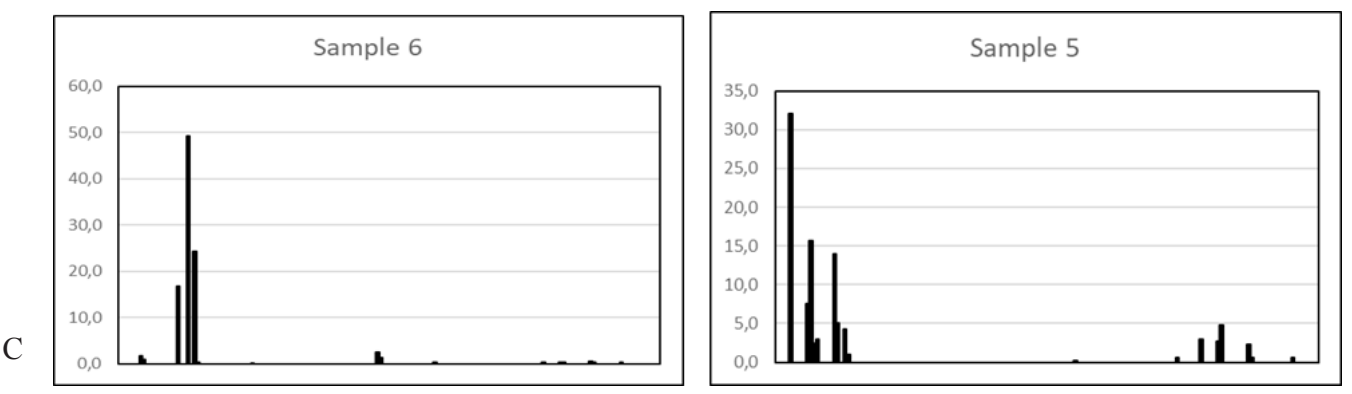

Figure 4 - Volatile organic compound profiles (A- type 1 samples: rich in hydrocarbons and ketones;

B- type 2 samples: rich in ketones and acids; $\mathbf{C}$ - type 3 samples: rich in acids

VOCs profile difference between bacilli and cocci starters. The PCA analysis of the VOCs groups showed an opposition between samples fermented with cocci and those fermented with bacilli starter along the second factor of the analysis (Figure 1). The discriminant analysis applied on the table (12 samples * 9 VOCs groups) showed that the mean profiles of each type of lactic acid bacteria can be discriminated with a percentage of well-classed at $83.3 \%$. The stepwise model showed that the main discriminating group of compounds was the group "others" molecules. The integration of other groups (the second discriminating parameter was ketones) did not improve the discriminating power. The variance analysis confirmed that only the group "other compounds" was significantly different $(\mathrm{P}<0.01)$ between the two types of lactic acid bacteria (Table 3). Variance analysis was then applied on the "Other VOCs" group only to identify the specific molecule explaining this difference between type of bacteria. It appeared that only one molecule differed significantly: indeed, oxime-methoxy-phenyl was significantly higher $(\mathrm{P}<0.01)$ in samples fermented with cocci bacteria $(5.9 \pm 4.5 \%)$ than with bacilli $(0.63 \pm 1.14 \%)$.

Table 3 - VOCs composition (in \%) of the fermented milk samples using Cocci or Bacilli starters

\begin{tabular}{|c|c|c|c|}
\hline Group of VOCs & Cocci & Bacilli & Pr $>$ F \\
\hline Acids & $43.01 \pm 16.1$ & $39.11 \pm 38.8$ & 0.838 \\
\hline Aldehydes & $1.8 \pm 1.8$ & $1.15 \pm 1.5$ & 0.520 \\
\hline Alcohol & $1.2 \pm 1.4$ & $6.45 \pm 6.9$ & 0.128 \\
\hline N-compounds & $6.4 \pm 6.1$ & $3.78 \pm 3.9$ & 0.357 \\
\hline Esters & $2.24 \pm 3.9$ & $1.74 \pm 2.1$ & 0.194 \\
\hline Aliphatic HC & $8.03 \pm 9.5$ & $22.15 \pm 20.9$ & 0.453 \\
\hline Aromatic HC & $5.9 \pm 4.7$ & $9.64 \pm 9.8$ & 0.090 \\
\hline Ketones & $21.3 \pm 10.3$ & $12.51 \pm 6.1$ & $0.009 *$ \\
\hline Others & $10.1 \pm 2.8$ & $3.48 \pm 3.9$ & 100 \\
\hline Total & 100 & & \\
\hline
\end{tabular}

The analysis of the full profiles (160 VOCs) by Mann-Whitney test showed that $77 \%$ of the molecules were significantly different between the two bacteria. However, after submission to discriminant analysis of the full table (12 samples, except control * 160 compounds), the combination of 3 molecules were sufficient to allow discriminating $100 \%$ of the samples. The main molecule was still oximemethoxy-phenyl. The second discriminant molecule was propanedioic acid, propyl- (group "Acids") and the third, 2-Propanamine (group "N-compound"). The values of the second molecule were $0.99 \pm 1.26$ and $3.44 \pm 3.18 \%$ for bacilli and cocci, respectively. For the third one, the values were $0.17 \pm 0.29$ and 0 respectively.

Variability in compounds population. Natural koumis is known to contain a large number of lactic bacteria and yeasts strains. For example, Bai and Ji 
(2016) [10] in China identified 55 LAB strains. Such microbiological biodiversity, due to spontaneous fermentation, could explain the high number of potential volatiles compounds in such typical beverages compared to our samples obtained after controlled fermentation. Compared to natural koumis which contained $5.8 \%$ ethanol, our samples of fermented milk had low quantity of alcohol. Indeed, the high level of ethanol usually found in natural koumis is usually due to yeasts [11] and not to bacteria as bacilli or cocci. However, three of our samples contained ethanol molecule (3.4\% in sample $9,1.3 \%$ in sample 11 and $0.9 \%$ in sample 13 ). It could be due to certain strains of bacteria (one sample was cocci and two, bacilli), but as the present study was based on preliminary data, the identification of strains was not yet achieved. Indeed, ethanol production could be possible in relatively high quantity with lactobacillus strains [12].

The use of cow milk as matrix for inoculation could also explain that only 5 compounds from natural koumis were common with the VOCs in our samples. Indeed, there is an important difference in lactose content between mare milk (60-80 g/L) and cow milk (30-50 g/L) and, consequently LAB strains could produce a higher compound variability.

Variability of VOCs profiles. Regarding the full VOCs profile, samples clearly divided into 3 types (issued from cluster analysis). The most apparent discriminant group of molecules was acid compounds. Control samples contained less acid compounds as they were not fermented. However, 3 samples of fermented milk presented similar pattern which attested the absence or very low level of hydrolysis of milk components. Probably, the strain of bacteria used in these samples (all bacilli) were not enough active in cow milk matrix or required more incubation time because a slow metabolic activity [13]. The second type of profile was characterized by its wide variety of compounds, with relatively higher proportion of Nand S-compounds (included in group "other"). Those molecules issued from hydrolyze of amino-acids and probably the bacteria strains (mostly cocci) in this VOCs profile type produced proteolytic enzymes. Ester compounds were also present in higher proportion which could be due mainly to fat hydrolysis [14]. The third type was milk with presence of ketones and overall high quantity of acids. Those samples were inoculated with bacilli strains probably able to have high acidification capacity. Bacilli strains are known to produce strong flavor in dairy products $[14 ; 15]$.

Discriminant parameters of lactic bacteria. Despite large number of aroma distinguishing VOCs profile of bacilli and cocci, 3 molecules only were involved in the discrimination. The most discriminating molecule (oxime-methoxy-phenyl-OMP) is a chemical product belonging to imine group which has sweetener taste used in the agro-industry [16]. OMP is normally largely present in UHT cow milk. Dursun, Güler and Şekerli (2017) [17] reported its proportion in VOCs profile of UHT milk at $46.07 \%$.

The second molecule was propanedioic acid, propyl-, an acid component for which no information was available. Despite its role to discriminate the two types of bacteria, its effects were not described in the literature.

At reverse, more information was available for the third discriminating parameter (2-propanamine). It is an amine, building block for several pesticides and herbicides. However, its proportion in our samples was quite low, and even nil in milk inoculated with cocci. The dissimilarity between bacilli and cocci is mainly morphological, but their fermentative activity appeared also quite distinct with two VOCS profiles with only $23 \%$ of the molecules having no significant difference. However, no data is available on the VOCs profile description for these two types of bacteria and their effect on flavor of dairy products.

\section{Conclusion}

The present study focused on VOCs profile of microflora from raw and fermented milk. The description of those profiles was never done previously. Yet, there is no description of the typical flavor of these products, popular in all Central Asia. The characterization of those flavor could be an essential element to describe the wide biodiversity of those traditional dairy beverages, the flavor of which depends on their microflora. With only 3 compounds, it was possible to distinguish the products inoculated by different types of bacteria with $100 \%$ probability. This means that VOCs profiles are highly dependent on the strains used for inoculation, which could have an important impact for agro-food industry to control fermentation process and then, to obtain new dairy products. For the next step, different matrices (milk from other species) for fermentation should be tested, and the strains of bacteria will be identified for a better understanding of their effect on flavor.

\section{Acknowledgements}

The present study was conducted in the framework of the project №037-16-ГК "Modernization of 
the test center for qualitative and quantitative studies of milk and dairy products" supported by "Science Fund" from the Ministry of Education and Science of the Republic of Kazakhstan.

\section{References}

1. Hansen A., Schieberle P. (2005) Generation of aroma compounds during sourdough fermentation: applied and fundamental aspects. Trends in Food Sci \& Tech., vol.16, no. 1-3, pp. 85-94.

2. Dragone G., Mussatto S.I., Oliveira J.M., Teixeira J.A. (2009) Characterisation of volatile compounds in an alcoholic beverage produced by whey fermentation. Food Chem., vol. 112, no. 4, pp.929-935.

3. Konuspayeva G., Faye B. (2011) Identité, vertus thérapeutiques et allégations santé: les produits laitiers fermentes d'Asie Centrale. Les cahiers de l'OCHA, vol. 15, pp. 35-145.

4. Seitov Z.S. (2005) Koumiss. Shubat, Almaty, $286 \mathrm{p}$.

5. Xu C.H., Chen G.S., Xiong Z.H., Fan Y.X., Wang X.C., Liu Y. (2016) Applications of solidphase microextraction in food analysis. TrAC Trends in Analyt Chem., vol. 80, pp. 12-29.

6. Jolliffe I. (2011) Principal component analysis. In: International encyclopedia of statistical science. Springer, Berlin, Heidelberg, pp. 1094-1096.

7. Longo M.A., Sanromán M.A. (2006) Production of food aroma compounds: microbial and enzymatic methodologies. Food Tech and Biotech., vol.44, no. 3, pp. 335-353.

8. Everitt B.S., Landau S., Leese M., Stahl D. (2011) Cluster analysis. John Wiley \& Sons Ltd., Chichester, p. 346.

9. Huberty C.J. (1994) Applied discriminant analysis. Wiley, University of Michigan, p. 466.
10. Bai L., Ji S. (2017) Isolation and identification of lactic acid bacteria from koumiss in Eastern Inner Mongolia of China. In: AIP Conference Proceedings, vol. 1794, no. 1, pp. 050005.1-050005.4.

11. Gadaga T.H., Viljoen B.C., Narvhus J.A. (2007) Volatile organic compounds in naturally fermented milk and milk fermented using yeasts, lactic acid bacteria and their combinations as starter cultures. Food Tech Biotech., vol. 45, no. 2, p. 195.

12. Pan D.D., Wu Z., Peng T., Zeng X.Q., Li H. (2014) Volatile organic compounds profile during milk fermentation by Lactobacillus pentosus and correlations between volatiles flavor and carbohydrate metabolism. J Dairy Sci., vol. 97, no. 2, pp. 624-631.

13. Mukisa I.M., Byaruhanga Y.B., Muyanja MBK.C., Langsrud T., Narvhus J.A. (2017) Production of organic flavor compounds by dominant lactic acid bacteria and yeasts from Obushera, a traditional sorghum malt fermented beverage. Food Sci Nutr., vol. 5, no. 3, pp. 702-712.

14. Chen C., Zhao S., Hao G., Yu H., Tian H., Zhao G. (2017) Role of lactic acid bacteria on the yogurt flavour: a review. Int J Food Prop., vol. 20, no. sup1, pp. s316-s330.

15. Dan T., Wang D., Wu S., Jin R., Ren W., Sun T. (2017) Profiles of Volatile Flavor Compounds in Milk Fermented with Different Proportional Combinations of Lactobacillus delbrueckii subsp. bulgaricus and Streptococcus thermophilus. Molecules, vol.22, no.10, p.1633.

16. Panten J., Surburg H. (2015) Flavors and Fragrances, 2. Aliphatic Compounds. In: Ullmann's Encyclopedia of Industrial Chemistry, Wiley-VCH, Weinheim, p. 55.

17. Dursun A., Güler Z., Emre Şekerli Y. (2017) Characterization of volatile compounds and organic acids in ultra-high-temperature milk packaged in tetra brik cartons. Int J Food Prop., vol.20, no.7, pp. 1511-1521. 